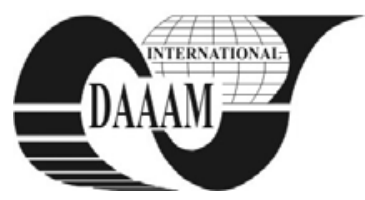

\title{
NUMERICAL METHODS OF THE EXAMINATION OF THE POSTURAL STABILITY
}

\author{
PIVNICKOVA, L[ucie]; VASEK, V[ladimir] \& DOLINAY, V[iliam]
}

\begin{abstract}
Posturography is a non-invasive medical technique used to evaluate vertigo. Traditional static posturography has several results, such as charts describing speed, direction, harmony of patient's tilts, but for the medical studies and formation of norms are more sufficient numerical outputs, which we are going to deal with in this paper. This article deals with numerical methods of calculation in evaluation of static posturography. Essential evaluations have been defined in the past, but still some significant signs remain hidden in charts or even in raw data. This paper show traditional numeric approaches and try to point the new one, focused on lateral deviation.
\end{abstract}

Key words: area, posturography, Romberg, vertigo, way

\section{INTRODUCTION}

Posturography is a non-invasive technique used to quantify the central nervous system adaptive mechanisms (sensory, motor and central) involved in the control of posture and balance, both in normal and abnormal conditions. Another term linked with posturography is vertigo. Vertigo is very common feeling, known more or less to everyone. Vertigo is not a disease, vertigo is symptom. Extensive studies on 30000 people of all age groups in Germany confirmed that about $20 \%$ men and up to $40 \%$ of adult women from time to time have feeling of motion when one is stationary. Vertigo may occur in all age groups, although with increasing age, the incidence increases (Rodder, 1990).

Posturography as an examination method is measuring of postural balance in static or dynamic conditions. Static methods are mostly judging standing balance, when is the patient placed in a standing posture on a fixed instrumented platform connected to sensitive detectors, which are able to detect the tiny oscillations of the body on the contrary dynamic methods generally used a special apparatus with a movable horizontal platform. (Vestibular Technologies, 2011)

This paper entirely deals with static posturography and its numerical evaluations.

\section{PROCESS OF EXAMINATION}

Static posturography is carried out by placing the patient in a standing posture on a fixed instrumented platform connected to sensitive detectors (force and movement transducers), which are able to detect the tiny oscillations of the body (Wiki, 2011). See Fig. 1.

The parameters of measurement:

(Based on common options for STP-03 platform)

- Measure period: $40 \mathrm{~ms}$

- $\quad$ Length of measure: $20 \mathrm{~s}$

- Area for calculating weighted area: $5^{\circ}$

- $\quad$ Angle step for display vectors: $15^{\circ}$

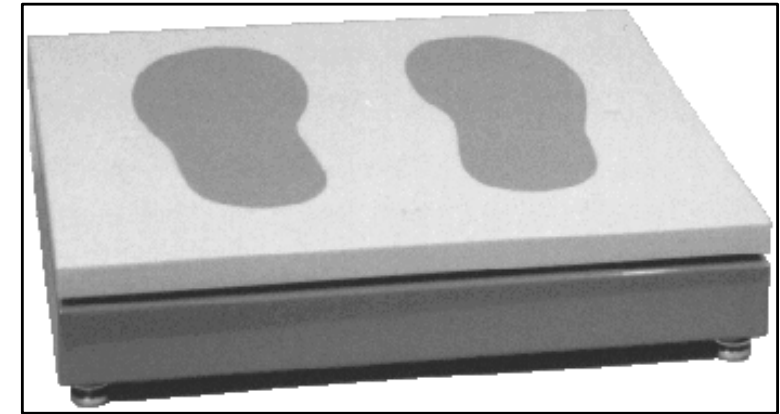

Fig. 1. Static posturography (Carreta s.r.o., 1998)

Traditional static posturography is based on Romberg test, which means that two exams are executed - with eyes open and then with eyes closed. The mutual relations between results are track. A normal person is upright, but the patient suffering from vertigo deviates from the vertical position as he (she) tries to compensate for a sense of movement that feels. The patient usually tilts to the side, which has affected labyrinth.

\section{MEASUREMENT RESULTS}

Posturograph STP-03 software (widely use in Czech Republic) calculates the value of the way (1) and area (2) of the patient center of gravity circumscribed over the posturographic platform. Evaluates the ratio between these values for the examination with open and closed eyes are important parts of vertigo assessment.

Parameter Way, $W(\mathrm{~cm} / \mathrm{s})$ describes the path of moving center of gravity, but because the examination time given a constant number, Way is characterized as the speed of center of gravity. Parameter Way for open eyes is marked with postfix $f$; $(W f)$ - visual fixation. Way for the closed eyes is marked with postfix s; (Ws) - visual suppression.

$$
\begin{gathered}
M_{i}=\sqrt{\left(x_{i+1}-x_{i}\right)^{2}+\left(y_{i+1}-y_{i}\right)^{2}} \\
W=\frac{T^{-1}}{n} \sum_{i=1}^{n} M_{i}[\mathrm{~mm} / \mathrm{s}]
\end{gathered}
$$

Where:

$$
\begin{array}{ll}
M_{i} & \text { particular element of way calculation } \\
T & \text { measure period [s] } \\
x, y & \text { center of gravity coordinates } \\
n & \text { number of measured samples }
\end{array}
$$

Parameter Area, A $\left(\mathrm{cm}^{2} / \mathrm{s}\right)$ indicates the area, which describes variation of center of gravity during the examination. Like the parameter Way, Area for the visual fixation is marked (Af) and for visual suppression (As). (Drsata, 2007) 


$$
\begin{gathered}
N_{i}=\frac{\left|\begin{array}{c}
\left(y_{i+1}-y_{0}\right) *\left(x_{i}-x_{0}\right) \\
-\left(y_{i}-y_{0}\right) *\left(x_{i+1}-x_{0}\right)
\end{array}\right|}{2} \\
A=\frac{1}{t} \sum_{i=1}^{n-1} N_{i}\left[\mathrm{~mm}^{2} / \mathrm{s}\right]
\end{gathered}
$$

Where:

$$
\begin{array}{ll}
N_{i} & \text { particular element of area calculation } \\
t & \text { length of measure [s] } \\
x_{0}, y_{0} & \text { average values of center of gravity coordinates }
\end{array}
$$
(3)

$$
\begin{gathered}
x_{0}=\frac{1}{n} \sum_{i=1}^{n} x_{i} \\
y_{0}=\frac{1}{n} \sum_{i=1}^{n} y_{i}
\end{gathered}
$$

\subsection{Visual presentation of results}

As mentioned earlier, the posturograph examination has also visual outputs. This paper is not dealing with those results directly so outputs will be describe shortly, but information covering by them are much more wider than used numerical results.

Fig. 2 shows center of gravity trajectories (patient body motion) focus and direction of motion vector evaluation. The above charts are for open eyes, bottom for closed eyes. On the left is the trajectory of motion, in the middle motion vectors in a bar-shaped view. On the right is shown the envelope vectors.

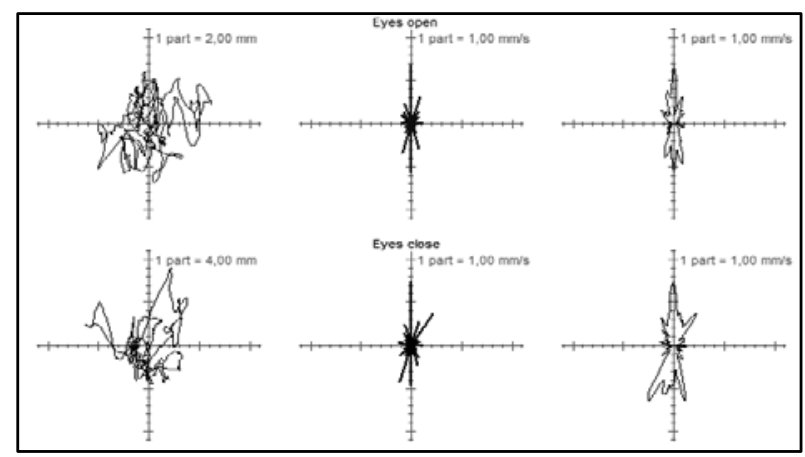

Fig. 2. Results in charts

Fig. 3 shows harmonic analysis of the patient's movements. The above charts are for open eyes bottom for closed eyes. On the left is the lateral component of movement, in the middle anteriopostural component of movement. On the right is the frequency analysis of the above mentioned components is showed. The area for the simulation analysis is highlighted by red line segments.

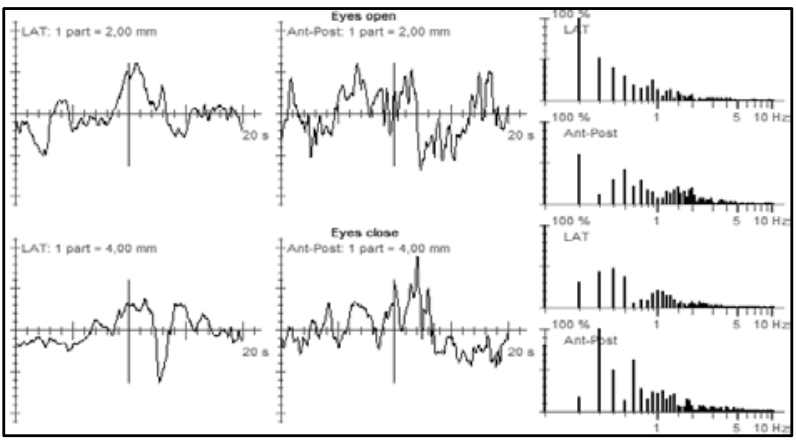

Fig. 3. Frequency analysis in charts

\subsection{Addition of side parameters}

For the vertigo evaluation it seems to be important to express the side and harmonic center of gravity move components. There is no direct numerical expression of these motion components, only visual presentation is partly covering it. We try to present simple procedure based on current data and nature of vertigo. Let's suppose, patients who have visual fixation, they have relatively good results but when loosing this fixation, the disturbance become wider. The same presumption can be applied to beginning of the examination and late course.

Modify the equation (3), replacing $n$ with $m$ where $m$ is the number of measured samples in the first second of the examination and retrieve new, average values of center of gravity coordinates $x_{b}$ and $y_{b}(4)$.

$$
\begin{aligned}
& x_{b}=\frac{1}{m} \sum_{i=1}^{m} x_{i} \\
& y_{b}=\frac{1}{m} \sum_{i=1}^{m} y_{i}
\end{aligned}
$$

Then the parameter (vector) $S$ can be defined (5).

$$
S=\overrightarrow{x_{b} x_{0}}(5)
$$

For the evaluation of dominant tilts is necessary to calculate orientation and vector size. These values expand current numerical results and improve diagnostics accuracy.

\section{CONCLUSION}

In summary, we aimed to current methods of posturography evaluation. The basic, widely used numerical and visual outputs were shown. This article also presents the new parameter in the form of vector $\mathrm{S}$ (side).

The parameter $\mathrm{S}$ seems to be important for expressing of the side deviation of the center of gravity in the vertigo evaluation. The result of the studies shown, for the time being, that it is not possible to aver its practical usability.

However, the proposed evaluation needs further study based on the verification from the practical use. Subsequently the rate of its practical usability will be determined.

The further research should also focus on the harmonicity of motion and its easy numerical presentation for standards formulation.

\section{ACKNOWLEDGEMENTS}

The work was supported by the European Regional Development Fund under the Project CEBIA-Tech No. CZ.1.05/2.1.00/03.0089.

The project "The methodology used to quantify and qualitative assessment of the postural balance" is supported by Internal Grant Agency of Tomas Bata University, IGA/47/FAI/11/D.

\section{REFERENCES}

Caretta s,r.o. (1998). Posturography, Available from: http://www.caretta.cz/software/posturograph/teorie.asp

Drsata, J. (2007). Computer posturography in the diagnosis and rehabilitation of vertiginous states, Hradec Kralove

LEJSKA, Mojmir (2010). Treatment of vertiginous states by vestibular rehabilitation, AUDIO-Fon centr Brno

Rodder, H.D. (1990). Der Schwindel. Arzte Zeitung Verlagsgesellschaft m.b.H., Neu-Isenburg

Vestibular Technologies, (2011). Posturography, Available from: http://www.vestibtech.com/Posturography.htm

***(2011) Posturography, Available from: http://en.wikipedia.org/wiki/Posturography 